\title{
Surrogate Based Optimization of Helicopter Rotor Blades for Vibration Reduction in Forward Flight
}

\author{
Bryan Glaz, Peretz P. Friedmann ${ }^{\dagger}$ and Li Liu ${ }^{\ddagger}$ \\ Department of Aerospace Engineering, The University of Michigan, Ann Arbor, MI, 48109, USA
}

The effectiveness of surrogate modeling of helicopter vibrations, and the use of the surrogates for optimization of helicopter vibration are studied. The accuracies of kriging, radial basis function interpolation, and polynomial regression surrogates are compared. In addition, the surrogates for the vibratory hub shears and moments are used to generate an objective function which is employed in an optimization study. The design variables consist of the cross-sectional dimensions of the structural member of the blade and the non-structural masses. The optimized blade is compared with a baseline rotor blade which resembles an MBB BO-105 blade. The results indicate that: (a) the kriging surrogates are the best for approximating vibratory hub loads over the entire design space and (b) and the surrogates can be used effectively in helicopter rotor vibration reduction studies.

\section{Nomenclature}

\begin{tabular}{ll}
$c$ & Blade chord \\
$C_{W}$ & Helicopter weight coefficient \\
$C_{d 0}$ & Blade profile drag coefficient \\
$C_{d f}$ & Flat plate drag coefficient \\
$\mathbf{D}$ & Vector of design variables \\
$\mathrm{E}$ & Young's modulus \\
$f(\mathbf{x})$ & Assumed polynomials which account for the 'global' behavior in kriging \\
$F_{4 X}, F_{4 Y}$, & \\
$F_{4 Z}$ & $4 /$ rev hub shears, non-dimensionalized by $m_{0} \Omega^{2} R^{2}$ \\
$\hat{F}_{4 X}, \hat{F}_{4 Y}$, & \\
$\hat{F}_{4 Z}$ & Surrogates for the non-dimensional $4 /$ rev hub shears \\
$\mathrm{g}(\mathbf{D})$ & Constraints \\
$\mathrm{h}$ & Height of the blade cross-section \\
$J$ & Objective function \\
$\hat{J}$ & Surrogate objective function \\
$J_{P}$ & Mass polar moment of inertia of the rotor \\
$m_{0}$ & Mass per unit length at the blade root \\
$m_{n s}$ & Non-structural mass located at the elastic axis \\
$M_{4 X}, M_{4 Y}$, & 4/rev hub moments, non-dimensionalized by $m_{0} \Omega^{2} R^{3}$ \\
$\hat{M}_{4 Z}$ & \\
$\hat{M}_{4 X}, \hat{M}_{4 Y}$, & Surrogates for the non-dimensional $4 /$ rev hub moments \\
$\hat{M}_{4 Z}$ & Number of rotor blades \\
$N_{b}$ & Number of behavior constraints \\
$N_{c}$ & Number of design variables \\
$N_{d v}$ & \\
\hline${ }^{*}$ Ph.D. Candidate, Student Member AIAA. \\
${ }^{\dagger}$ François-Xavier Bagnoud Professor, Fellow AIAa, AHs \\
${ }^{\ddagger}$ Postdoctoral Researcher, Member AIAA.
\end{tabular}




\begin{tabular}{|c|c|}
\hline$N_{s p}$ & Number of sample points \\
\hline$N_{t p}$ & Number of test points \\
\hline$p_{k}, \vartheta_{k}$ & Fitting parameters in kriging corresponding to the $k^{t h}$ design variable \\
\hline$R$ & Blade radius \\
\hline $\mathbf{R}_{\mathrm{krg}}$ & Spatial correlation matrix used in kriging \\
\hline$R_{\mathrm{krg}}(\cdot)$ & Spatial correlation function in kriging \\
\hline $\mathbf{r}_{\mathrm{krg}}(\mathbf{x})$ & Spatial correlation vector in kriging \\
\hline$t_{1}, t_{2}, t_{3}$ & Thicknesses of the blade cross-section, see Fig. 3 \\
\hline $\mathbf{w}$ & Vector of coefficients in RBF interpolation, with elements $w_{i}$ \\
\hline $\mathbf{x}^{(i)}$ & $i^{\text {th }}$ sample point \\
\hline$x_{1}, x_{2}$ & Cross-sectional dimensions, see Fig. 3 \\
\hline$X_{F A}, Z_{F A}$ & $\begin{array}{l}\text { Longitudinal and vertical offsets between rotor hub and helicopter aerodynamic center, see } \\
\text { Fig. } 6\end{array}$ \\
\hline$X_{F C}, Z_{F C}$ & $\begin{array}{l}\text { Longitudinal and vertical offsets between rotor hub and helicopter center of gravity, see } \\
\text { Fig. } 6\end{array}$ \\
\hline$y(\mathbf{x})$ & Unknown function to be approximated \\
\hline$y^{(i)}$ & output response at $\mathbf{x}^{(i)}$ \\
\hline $\mathbf{y}$ & Vector of observed function outputs \\
\hline$\hat{y}(\mathbf{x})$ & Approximation of $y(\mathbf{x})$ \\
\hline$Z(\mathbf{x})$ & Realization of a stochastic process in kriging \\
\hline$\alpha_{d}$ & Flight descent angle, see Fig. 6 \\
\hline$\beta$ & Constant used in kriging \\
\hline$\hat{\beta}$ & Generalized least squares estimate of $\beta$ \\
\hline$\beta_{0}, \beta_{i}, \beta_{i j}$ & Fitting coefficients in polynomial regression \\
\hline$\beta_{p}$ & Blade precone angle \\
\hline$\epsilon_{\mathrm{pr}}$ & Approximation error in polynomial regression \\
\hline$\varepsilon$ & Absolute percent error of surrogate predictions \\
\hline$\lambda_{k}$ & Hover stability eigenvalue for $k^{t h}$ mode \\
\hline$\zeta_{k}, \omega_{k}$ & Real and imaginary parts of $\lambda_{k}$, respectively \\
\hline$\mu$ & Advance ratio \\
\hline$\nu$ & Poisson's ratio \\
\hline$\Omega$ & Rotor angular speed \\
\hline$\omega_{F 1}, \omega_{L 1}, \omega_{T 1}$ & Fundamental rotating flap, lead-lag and torsional frequencies, /rev \\
\hline$\omega_{L}, \omega_{U}$ & Lower and upper bounds for frequency constraints, /rev \\
\hline$\phi_{\mathrm{RBF}}(\cdot)$ & Spatial correlation function in RBF interpolation \\
\hline$\Phi_{\mathrm{RBF}}$ & Spatial correlation matrix in RBF interpolation \\
\hline$\phi_{\mathrm{RBF}}(\mathbf{x})$ & Spatial correlation vector in RBF interpolation \\
\hline$\rho_{\text {filler }}$ & Material density for non-structural filler mass \\
\hline$\rho_{\text {struct }}$ & Material density for the structural member of the blade \\
\hline$\sigma$ & Rotor solidity \\
\hline$\sigma_{v a r}^{2}$ & Variance of the Gaussian process $Z(\mathbf{x})$ \\
\hline$\hat{\sigma}_{v a r}^{2}$ & Generalized least squares estimate of $\sigma_{v a r}^{2}$ \\
\hline$\tau$ & Fitting parameter in RBF interpolation \\
\hline$\theta_{\mathrm{pt}}$ & Blade built-in pre-twist angle \\
\hline
\end{tabular}

\section{Introduction}

VIBration is one of the most critical concerns in the design of modern rotorcraft. Stricter demands for enhanced performance, comfort, and customer acceptance require designs with reduced vibration levels. In helicopters, the dominant source of vibrations is the rotor, which transfers vibrations to the rotor hub and fuselage at harmonics that are predominantly $N_{b} / \mathrm{rev}$, where $N_{b}$ is the number of blades.

During the last 25 years, two principal approaches to vibration reduction have emerged. The first approach is passive and uses structural/multidisciplinary optimization for reducing vibrations, ${ }^{1-4}$ while the second approach utilizes active control methods. ${ }^{5,6}$ This paper focuses on the passive approach. In the passive approach the vibration reduction problem is formulated as a mathematical optimization problem 
subject to appropriate constraints. The objective function consists of a suitable combination of the $N_{b} / \mathrm{rev}$ hub shears and moments that are computed from an aeroelastic response code; the constraints are blade stability margins, frequency constraints, an autorotation constraint, and constraints associated with the blade geometry. The design variables can be dimensions of the blade cross-section, mass and stiffness distributions along the span, or geometrical parameters which define advance geometry tips. Typical levels of vibration reduction achieved with passive approaches have been in the range of 50-60\%.

Due to the complex aerodynamic environment in the rotary-wing problem, aeroelastic response simulations needed for vibratory load calculations are computationally expensive and therefore numerous evaluations of the vibration objective function are costly. Therefore, direct combination of the objective function generated by the aeroelastic response simulation with traditional optimization algorithms is computationally very expensive. Moreover, traditional optimization search algorithms can converge to local optima, which are known to occur in this class of problems.

To overcome these obstacles, approximation concepts have been used. A widely used approach to approximating the vibration objective function and constraints is to use Taylor series expansions about local design points. ${ }^{7}$ The derivatives needed for the Taylor series are calculated using difference formulas, or analytical sensitivity derivatives. These approximations to the objective function and constraints are used to replace the actual problem with an approximate one that is used in conjunction with the optimizer to obtain an optimal design. This method was used in Refs. 2 and 8 to study vibration reduction of composite rotor blades with advanced geometry tips in forward flight. The disadvantages of this method are that it is a local approximation in the vicinity of a design point and a local search procedure is used. Even when augmenting this method with move limits or a trust region strategy, ${ }^{9,10}$ convergence is only guaranteed to a local optimum.

An alternative to the local Taylor expansion method is to use global approximation methods; i.e. methods which try to capture the behavior of a function over the entire design space. In one such vibration reduction study, Ganguli ${ }^{11}$ used a $2^{\text {nd }}$ order polynomial global approximation of the vibration objective function and obtained $30 \%$ vibration reduction. The $2^{\text {nd }}$ order polynomial was found to be accurate only in the vicinity of the baseline design. In addition to polynomials, there is a class of global approximation methods based on a stochastic process, which yield probabilistic measures of the uncertainty in the approximation (or surrogate) and can be used in a global search of the design space. ${ }^{12}$ The advantages of surrogate-based optimization (SBO) with stochastic process based global approximations are threefold: fewer "true" function evaluations (thus fewer expensive aeroelastic simulations), the formulation is conducive to parallel computing, and facilitation of a more "global" search of the design space. Illustrative examples of the benefits of SBO can be found in the studies by Sóbester et al. ${ }^{13}$ and Booker et al. ${ }^{14}$

In Ref. 13, surrogate methods and conventional methods - i.e. no approximations of the objective function combined with genetic algorithms and gradient based search methods - were used to find the optima of closed form five-dimensional test functions. The test functions were not computationally expensive to evaluate, and were only used for the purposes of testing the surrogate based optimization (SBO) method. In addition, Sóbester et al. took advantage of the opportunities for parallel computing afforded by SBO. Not only did SBO converge to the optima in less time, but the optimum yielded by SBO was superior to the optimum obtained from conventional optimization algorithms which do not use approximations of the objective function. Sóbester employed a stochastic process based approximation method known as radial basis function (RBF) interpolation.

In Ref. 14, surrogate methods were applied to minimization of helicopter vibration, using as few as 10 and as many as 56 design variables to characterize the rotor blade. The cross-sectional design variables were mass, center of gravity offset from the elastic axis, and the blade stiffnesses. The analysis code Tech01 ${ }^{15}$ was used to generate hub shears and moments. In Ref. 14, the stochastic process based method known as kriging interpolation was used to approximate the objective function. A more optimal design compared to results obtained using conventional search methods (genetic algorithms, for example) was achieved, and with fewer objective function evaluations. It is important to note that the principal focus of Ref. 14 was the effectiveness of surrogate objective functions, and therefore accurate modeling of the aerodynamic environment of a rotor blade during flight was not considered to be important. Consequently, accurate free wake models were sacrificed for a computationally less expensive prescribed wake model. Thus, the model of the helicopter vibratory loads was not sufficiently reliable to produce a realistic blade design.

An important feature of the surrogate based optimization approach using interpolation is that the method is computationally effective when the number of design variables is relatively small (less than 50). Fortunately, 
this is compatible with helicopter rotor blade optimization problems where the number of important design variables can be limited to this range. The overall objective of this paper is to examine the applicability of global approximation methods to the rotor blade vibration reduction problem in forward flight. To achieve this objective, the suitability of three methods for generating approximate representations of the problem are considered:

1. Polynomial Regression

2. Kriging

3. Radial basis functions (RBF's)

The effectiveness of these methods is compared by considering a rotor vibration reduction problem in forward flight.

\section{Overview of the Aeroelastic Response and Stability Analysis}

The simulation code used in this study is based on a comprehensive aeroelastic analysis code. ${ }^{6,16-21}$ The aeroelastic response analysis can represent the behavior of hingeless rotor blades as shown in Fig. 1, with actively controlled flaps; as well as blades with advanced geometry tips shown in Fig. 2. The key ingredients of the aeroelastic response analysis are: (1) the structural dynamic model, (2) the unsteady aerodynamic model and (3) a coupled trim/aeroelastic response procedure that is required for the computation of the steady state blade response. The aeroelastic response analysis and an overview of the aeroelastic stability in hover calculation are described next.

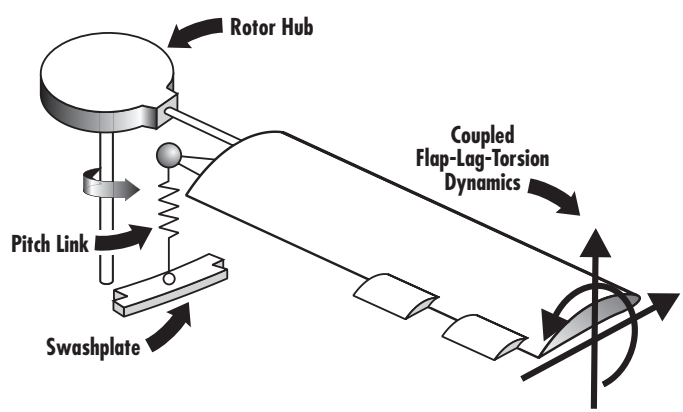

Figure 1. Helicopter rotor blade with trailing edge flaps.

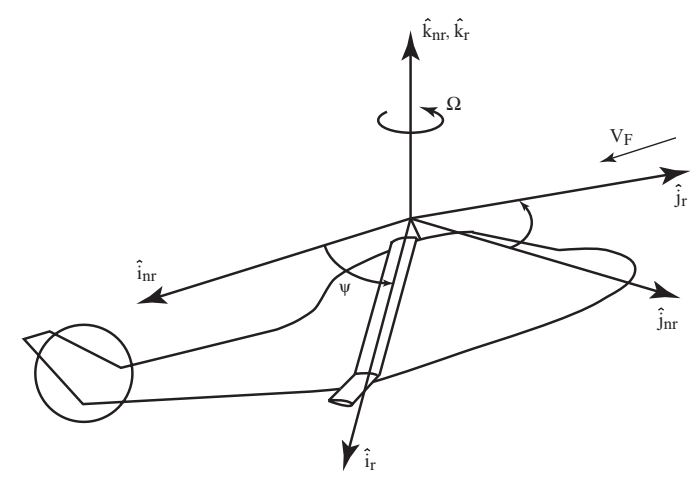

Figure 2. A blade with advanced geometry tip.

\section{A. Structural Dynamic Model}

The structural dynamic model is based on an analysis developed by Yuan and Friedmann ${ }^{2,8}$ which is capable of modeling composite blades with transverse shear deformations, cross-sectional warping, and swept tips. This study is limited to the behavior of isotropic blades with spanwise varying properties. The equations of motion are formulated using a finite element discretization of Hamilton's principle, with the assumption that the blade undergoes moderate deflections. The beam type finite elements used for the discretization have 23 nodal degrees of freedom. Normal modes are used to reduce the number of structural degrees of freedom. In this study, eight modes are used: the first 3 flap modes, first 2 lead-lag modes, first 2 torsional modes, and the first axial mode.

\section{B. Aerodynamic Model}

The attached flow blade section aerodynamics are calculated using a rational function approach (RFA). ${ }^{18,22}$ The RFA approach is a two-dimensional unsteady time-domain theory that accounts for compressibility as well as variations in the oncoming flow velocity. This two-dimensional aerodynamic model is linked to 
an enhanced free-wake model which provides a non-uniform inflow distribution at closely spaced azimuthal steps. ${ }^{23-25}$ Although the simulation code can also account for dynamic stall at high advance ratios, ${ }^{20}$ dynamic stall was not considered in this paper because the vibration levels being approximated are those due to blade vortex interaction (BVI), which occurs at low advance ratios.

\section{Coupled Trim/Aeroelastic Response}

The combined structural and aerodynamic equations form a system of coupled ordinary differential equations that are cast into first order state variable form ${ }^{18}$ and integrated in the time domain using the AdamsBashforth predictor-corrector algorithm. A propulsive trim procedure, where six equilibrium equations (three forces and three moments) are enforced, is used in this study. ${ }^{16,26}$ The trim equations are solved in a coupled manner with the aeroelastic equations of motion. The vibratory hub shears and moments are found by integrating the distributed inertial and aerodynamic loads over the entire blade span in the rotating frame, then transforming these loads to the hub-fixed non-rotating system, and summing the contributions from each blade. ${ }^{8}$ In the process, cancellation of various terms occurs and the primary components of the hub shears and moments have frequency of $N_{b} / \mathrm{rev}$, which is known as the blade passage frequency.

\section{Aeroelastic Stability in Hover}

The process for determining the hover stability of the blade is similar to that used by Yuan and Friedmann, ${ }^{8}$ and is described below:

1. The non-linear static equilibrium solution of the blade is found for a given pitch setting and uniform inflow, by solving a set of nonlinear algebraic equations. Note that uniform inflow is used only in the hover stability calculation. The forward flight analysis employs a free-wake model for inflow calculation.

2. The governing system of ordinary differential equations are linearized about the static equilibrium solution by writing perturbation equations and neglecting second-order and higher terms in the perturbed quantities. The linearized equations are rewritten in first-order state variable form.

3. The real parts of the eigenvalues of the first-order state variable matrix, $\lambda_{k}=\zeta_{k}+i \omega_{k}$, determine the stability of the system. If $\zeta_{k} \leq 0$ for all $k$, the system is stable.

The linearization process in Ref. 8 is modified to account for the aerodynamic states introduced by the RFA model. Details on the linearization process with RFA aerodynamics are provided in Appendix A.

\section{Formulation of the Blade Optimization Problem}

The formulation of the blade optimization problem in forward flight consists of several ingredients: the objective function, design variables, and constraints. The mathematical formulation of the optimization is stated below.

Find the vector of design variables $\mathbf{D}$ which minimizes the objective function, i.e. $J(\mathbf{D}) \rightarrow$ min, where the objective function consists of a combination of the $N_{b} /$ rev oscillatory hub shears and moments. For a four bladed rotor, the objective function is given by

$$
J=K_{S} \sqrt{\left(F_{4 X}\right)^{2}+\left(F_{4 Y}\right)^{2}+\left(F_{4 Z}\right)^{2}}+K_{M} \sqrt{\left(M_{4 X}\right)^{2}+\left(M_{4 Y}\right)^{2}+\left(M_{4 Z}\right)^{2}}
$$

where $K_{S}$ and $K_{M}$ are appropriately selected weighting factors.

The vector of design variables $\mathbf{D}$ consists of the thicknesses $t_{1}, t_{2}, t_{3}$, and the non-structural mass $m_{n s}$ located on the elastic axis (shear center) shown in Fig. 3 and specified at several spanwise locations. The design variables have side constraints to prevent them from reaching impractical values; these are stated as

$$
\mathbf{D}_{j}^{(L)} \leq \mathbf{D} \leq \mathbf{D}_{j}^{(U)}, \quad j=1,2, \ldots, N_{d v} .
$$

In addition, three types of behavior constraints, given by

$$
g_{i}(\mathbf{D}) \leq 0, \quad i=1,2, \ldots, N_{c},
$$




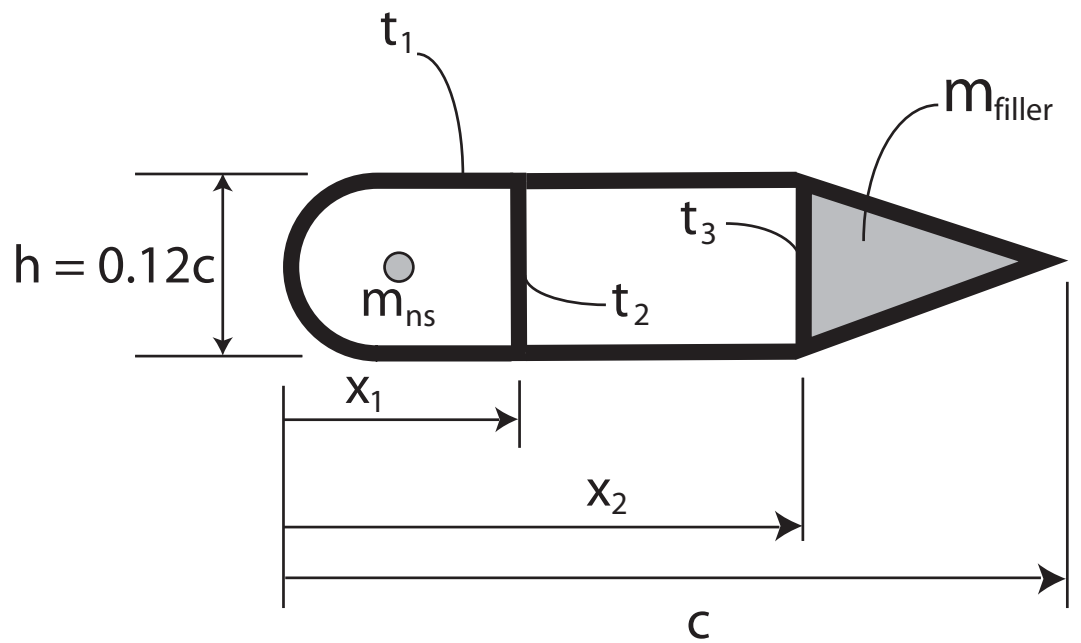

Figure 3. Simplified model of the blade structural member.

are placed on the design variables. The first type of behavior constraints are frequency placement constraints, which are prescribed upper and lower bounds on the fundamental flap, lag, and torsional frequencies of the blade. The frequency placement constraints on the fundamental flap frequency are written as

$$
\mathrm{g}_{\text {flap }}(\mathbf{D})=\frac{\omega_{F 1}}{\omega_{U}}-1 \leq 0
$$

and

$$
\mathrm{g}_{\text {flap }}(\mathbf{D})=1-\frac{\omega_{F 1}}{\omega_{L}} \leq 0
$$

where $\omega_{U}$ and $\omega_{L}$ are the prescribed upper and lower bounds on the fundamental flap frequency. Similar constraints are placed on the lag and torsional frequencies, i.e. $\mathrm{g}_{\text {lag }}$ and $\mathrm{g}_{\text {torsion. }}$ In addition, all blade frequencies must differ from integer multiples of the angular velocity $-1 / \mathrm{rev}, 2 / \mathrm{rev}, 3 / \mathrm{rev}, \ldots$, etc. - to avoid undesirable resonances.

Another behavior constraint is an autorotational constraint, which ensures that mass redistributions produced during the optimization do not degrade the autorotational properties of the rotor. Although there are several indices which can be used to represent the autorotational properties of the blade, the one used in this study is to require that the mass polar moment of inertia of the rotor be at least $90 \%$ of its baseline value. ${ }^{27}$ Mathematically, this is expressed as

$$
\mathrm{g}(\mathbf{D})=1-\frac{J_{P}}{0.9 J_{P 0}} \leq 0
$$

where $J_{P}$ is the mass polar moment of inertia of the rotor when it is spinning about the shaft, and $J_{P 0}$ is the baseline value.

The last behavior constraints are aeroelastic stability margin constraints, expressed mathematically as

$$
\mathrm{g}_{k}(\mathbf{D})=\zeta_{k}+\left(\zeta_{k}\right)_{\min } \leq 0, \quad k=1,2, \ldots, N_{m}
$$

where $N_{m}$ is the number of normal modes, $\zeta_{k}$ is the real part of the hover eigenvalue for the $k^{t h}$ mode, and $\left(\zeta_{k}\right)_{\min }$ is the minimum acceptable damping level for the $k_{t h}$ mode. It should be noted that the most critical modes for stability are usually the first and second lag modes.

\section{Global Approximation Methods}

The goal in using global approximation, or surrogate, methods is to replace the true objective function with smooth functional relationships of acceptable accuracy that can be evaluated quickly. In order to construct the surrogate, the objective function must first be evaluated over a set of design points. The 
surrogate is then generated by fitting the initial design points. Although function evaluations, which come from the expensive helicopter simulations, are needed to form the approximation, the initial investment of computer time associated with function evaluations is significantly reduced compared to global searches using non-surrogate based optimization methods. Once the surrogates have been obtained, they are used to replace the more expensive "true" objective function in the search for the optimum. In this study, the vibratory hub shears and moments in Eq. 1 are replaced by surrogates, which results in an approximate objective function expressed as

$$
\hat{J}=K_{S} \sqrt{\left(\hat{F}_{4 X}\right)^{2}+\left(\hat{F}_{4 Y}\right)^{2}+\left(\hat{F}_{4 Z}\right)^{2}}+K_{M} \sqrt{\left(\hat{M}_{4 X}\right)^{2}+\left(\hat{M}_{4 Y}\right)^{2}+\left(\hat{M}_{4 Z}\right)^{2}} .
$$

Descriptions of several methods for constructing such approximations are given below.

\section{A. Design of Computer Experiments}

When the initial data set is produced by a deterministic computer code (as is the case in the vibration reduction problem), the term "design of computer experiments," is more appropriate than design of experiments. $^{28,29}$ The distinction is necessary because in physical experiments there is measurement error and other random sources of noise that cannot be controlled, and this affects the choice of the design point. However, in computer experiments, there is no random error; i.e., for a deterministic computer code, a given input will always yield the same output. Thus, the design of computer experiments need only be space-filling. Figure 4 illustrates the difference between a conventional design of experiment and a space-filling design. In the figure, locations of design points where experiments are to be conducted, which in this case represent design points where aeroelastic response simulations are performed, are illustrated for a design space which has two design variables.
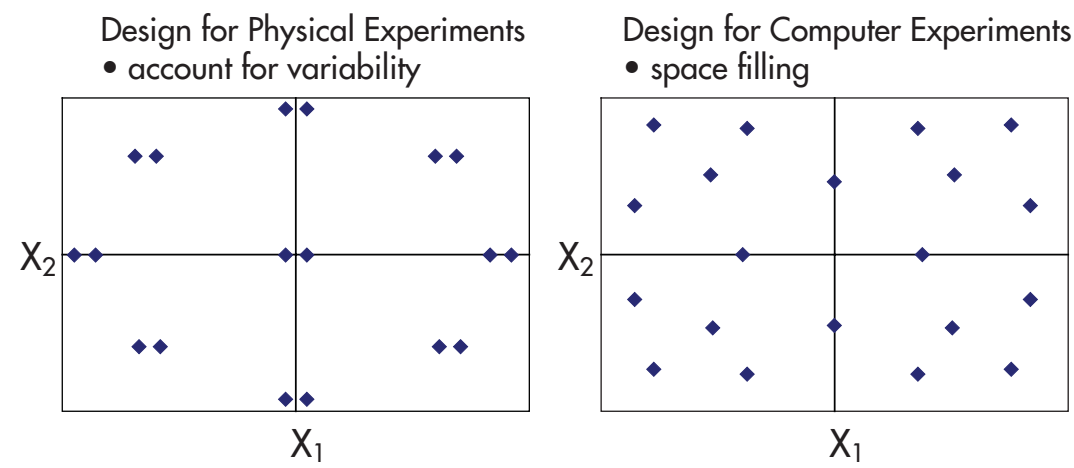

Figure 4. Design of physical experiment vs. design of computer experiment

A commonly used space-filling design is Latin hypercube sampling (LHS). ${ }^{30}$ In LHS, each design variable is partitioned into $N_{s p}$ equally spaced sections, or strata. Every design variable $D_{i}$, where $i=1,2, \ldots, N_{d v}$, is sampled once in each strata, which forms $N_{d v}$ vectors of size $N_{s p}$. The components of the $N_{d v}$ vectors are then randomly combined to form an $N_{s p} \times N_{d v}$ matrix known as a Latin hypercube, where each row corresponds to a design point at which a computer experiment is performed. A major disadvantage of Latin hypercube sampling is that design points can cluster together due to the random process by which design points are created. To prevent this, Optimal Latin hypercube $(\mathrm{OLH})^{31}$ sampling is used in this study to ensure a more uniform (or space-filling) design of computer experiment. Optimal Latin hypercube sampling creates a more uniform design than conventional LHS by maximizing a spreading criteria, rather than randomly creating design points from the samples. Details on the OLH algorithm used in the present study can be found in Ref. 32. Figure 5 illustrates the difference between a conventional Latin hypercube and an optimal Latin hypercube.

Methods for fitting the data points in the OLH are described next.

\section{B. Polynomial Regression}

Suppose a deterministic function of $N_{d v}$ design variables, that needs to be approximated, has been evaluated at $N_{s p}$ sample points. Sample point $i$ is denoted $\mathbf{x}^{(i)}=\left(x_{1}^{(i)}, \ldots, x_{N_{d v}}^{(i)}\right)$ and the associated response is given 

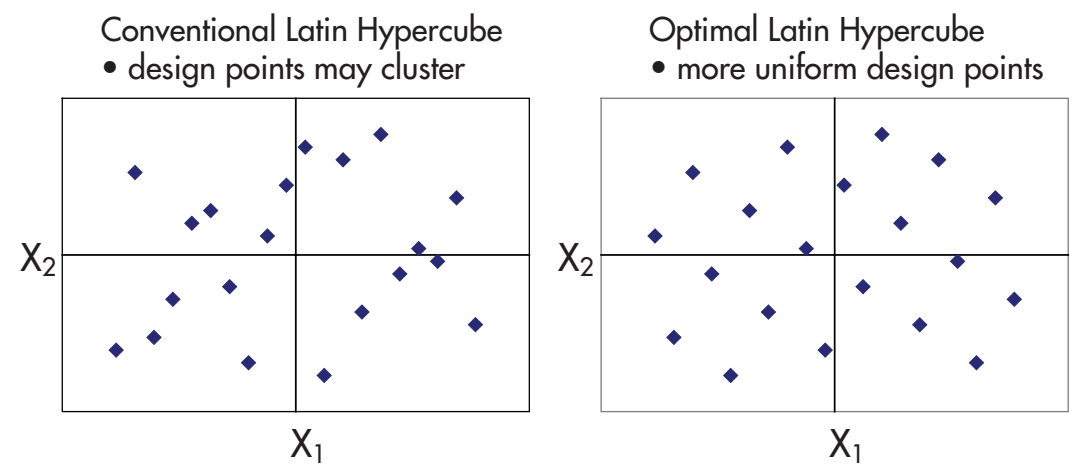

Figure 5. Conventional LH vs. Optimal LH in two dimensional design space.

by $y^{(i)}=y\left(\mathbf{x}^{(i)}\right)$ for $i=1, \ldots, N_{s p}$. A polynomial regression approximation to $y(\mathbf{x})$ can be written as

$$
y(\mathbf{x})=\hat{y}(\mathbf{x})+\epsilon_{\mathrm{pr}}
$$

where $\hat{y}(\mathbf{x})$ is the function chosen to approximate the true response $y(\mathbf{x})$, and $\epsilon_{\mathrm{pr}}$ is the error associated with the approximation. It is important to note that the errors are assumed to be independent; i.e. the errors at two points close together will not necessarily be close. This assumption will be revisited when considering kriging. In this study, $2^{\text {nd }}$ order polynomials are used for $\hat{y}(\mathbf{x})$. The least squares regression approximation is given as $^{33}$

$$
\hat{y}_{p r}=\beta_{0}+\sum_{i=1}^{N_{d v}} \beta_{i} x_{i}+\sum_{i=1}^{N_{d v}} \sum_{j=1, i<j}^{N_{d v}} \beta_{i j} x_{i} x_{j}+\sum_{i=1}^{N_{d v}} \beta_{i i} x_{i}^{2} .
$$

\section{Kriging}

Kriging is based on the fundamental assumption that errors are correlated, which is in contrast to the assumption of independent or uncorrelated errors made in polynomial regression. This implies that one assumes the errors at two points close together will be close. In fact, the assumption that the errors are uncorrelated is only appropriate when the sources of error are random, such as in the case of measurement error or noise. In the case of deterministic computer simulations, there is no source of random error. Therefore, it is more reasonable to assume that the error terms will be correlated and that this correlation is higher the closer two points are to each other. In kriging, the unknown function $y(\mathbf{x})$ is assumed to be of the form

$$
y(\mathbf{x})=f(\mathbf{x})+Z(\mathbf{x})
$$

where $f(\mathbf{x})$ is an assumed function (usually polynomial form) and $Z(\mathbf{x})$ is a realization of a stochastic (random) process which is assumed to be a Gaussian process with zero mean and variance of $\sigma_{\text {var }}^{2}$ (i.e. $Z(\mathbf{x})$ follows a normal, or Gaussian, distribution). ${ }^{12,34}$ The function $f(\mathbf{x})$ can be thought of as a global approximation of $y(\mathbf{x})$, while $Z(\mathbf{x})$ accounts for local deviations which ensure that the kriging model interpolates the data. The covariance matrix of $Z(\mathbf{x})$, which is a measure of how strongly correlated two points are, is given by

$$
\operatorname{Cov}\left[Z\left(\mathbf{x}^{(i)}\right), Z\left(\mathbf{x}^{(j)}\right)\right]=\sigma_{v a r}^{2} \mathbf{R}_{\mathrm{krg}}
$$

where each element of the $N_{s p} \times N_{s p}$ correlation matrix $\mathbf{R}_{\mathrm{krg}}$ is given by

$$
\left(R_{\mathrm{krg}}\right)_{i j}=R_{\mathrm{krg}}\left(\mathbf{x}^{(i)}, \mathbf{x}^{(j)}\right)
$$

and $R_{\mathrm{krg}}\left(\mathbf{x}_{i}, \mathbf{x}_{j}\right)$ is a correlation function which accounts for the effect of each interpolation point on every other interpolation point. This function is called the spatial correlation function (SCF) and is chosen by the user. The most commonly used SCF is the Gaussian correlation function,

$$
R_{\mathrm{krg}}\left(\mathbf{x}^{(i)}, \mathbf{x}^{(j)}\right)=\exp \left[-\sum_{k=1}^{N_{d v}} \vartheta_{k}\left|x_{k}^{(i)}-x_{k}{ }^{(j)}\right|^{p_{k}}\right],
$$


which is also employed in this study. The Gaussian SCF is dependent on the distance between two points. As two points move closer to each other, $\left|x_{k}{ }^{(i)}-x_{k}{ }^{(j)}\right| \rightarrow 0$ and Eq. 14 approaches unity, which is the maximum value of the Gaussian SCF. In other words, the Gaussian SCF recovers the intuitive property that the closer two points are to each other, the higher the correlation between the points.

The fitting parameters $\vartheta_{k}$ and $p_{k}$ are unknown correlation parameters which need to be determined. In order to determine these parameters, the form of $f(\mathbf{x})$ needs to chosen. The most common choice for $f(\mathbf{x})$ is $f(\mathbf{x})=\beta$ where $\beta$ is a constant. Previous studies have found that modeling with the SCF is so effective, that using a constant for the global behavior results in little loss of fidelity. ${ }^{12,28,34,35}$ Another common simplification, is to fix all $p_{k}=2$. When this simplification is combined with the constant global approximation, the approximation method is known as ordinary kriging. In the present study, kriging models where $p_{k}$ are not fixed at 2 will be compared with ordinary kriging models. In order to find $\vartheta_{k}$ and $p_{k}$, the generalized least square estimates of $\beta$ and $\sigma_{v a r}^{2}$, denoted by $\hat{\beta}$ and $\hat{\sigma}_{v a r}^{2}$ respectively, are employed: ${ }^{2,34}$

$$
\hat{\beta}=\left(\mathbf{1}^{\mathrm{T}}\left(\mathbf{R}_{\mathrm{krg}}\right)^{-1} \mathbf{1}\right)^{-1} \mathbf{1}^{\mathrm{T}}\left(\mathbf{R}_{\mathrm{krg}}\right)^{-1} \mathbf{y}
$$

and

$$
\hat{\sigma}_{\text {var }}^{2}=\frac{(\mathbf{y}-\mathbf{1} \hat{\beta})^{\mathrm{T}}\left(\mathbf{R}_{\mathrm{krg}}\right)^{-1}(\mathbf{y}-\mathbf{1} \hat{\beta})}{N_{s p}}
$$

where $\mathbf{1}$ is a vector populated by ones and $\mathbf{y}$ is a vector of observed function outputs at the interpolation points; both vectors are of length $N_{s p}$. With $\hat{\sigma}_{v a r}^{2}$ and $\hat{\beta}$ known, $\vartheta_{k}$ and $p_{k}$ are found such that a likelihood function $^{12,34,36}$ is maximized. The likelihood function, given in Eq. 17, is a measure of the probability of the sample data (or interpolation data) being drawn from a probability density function associated with a Gaussian process. Since the stochastic process associated with kriging has been assumed to be a Gaussian process, one seeks the set of $\vartheta_{k}$ and $p_{k}$ that maximize the probability that the interpolation points have been drawn from a Gaussian process.

$$
-\frac{\left[N_{s p} \ln \left(\hat{\sigma}_{v a r}^{2}\right)+\ln \left|\mathbf{R}_{\mathrm{krg}}\right|\right]}{2}
$$

The maximum likelihood estimates (MLE's) of $\vartheta_{k}$ and $p_{k}$ represent the "best guesses" of the fitting parameters. Any values of $\vartheta_{k}$ and $p_{k}$ would result in an interpolating surrogate, but the "best" kriging surrogate is found by optimizing the likelihood function. This auxiliary optimization process can result in significant fitting time depending on the size of the system. Due to the optimization process needed to create the kriging surrogate, kriging is only appropriate when the time needed to generate the interpolation points is much larger than the time to interpolate the data - which is the case in the helicopter vibration problem. The UMDIRECT global optimization algorithm ${ }^{34,37}$ is used to find the optimal set of the fitting parameters. With all parameters known, the kriging approximation to a function $y(\mathbf{x})$ can be written as ${ }^{12,28,34,35}$

$$
\hat{y}_{\mathrm{krg}}=\hat{\beta}+\mathbf{r}_{\mathrm{krg}}(\mathbf{x})^{\mathrm{T}}\left(\mathbf{R}_{\mathrm{krg}}\right)^{-1}(\mathbf{y}-\mathbf{1} \hat{\beta})
$$

where

$$
\mathbf{r}_{\mathrm{krg}}(\mathbf{x})=\left[R_{\mathrm{krg}}\left(\mathbf{x}, \mathbf{x}^{(1)}\right), R_{\mathrm{krg}}\left(\mathbf{x}, \mathbf{x}^{(2)}\right), \ldots, R_{\mathrm{krg}}\left(\mathbf{x}, \mathbf{x}^{\left(N_{s p}\right)}\right)\right]^{\mathrm{T}}
$$

The column vector $\mathbf{r}_{\mathrm{krg}}(\mathbf{x})$ of length $N_{s p}$ is the correlation vector between an arbitrary point $\mathbf{x}$ and the interpolation points, $\mathbf{x}^{(1)}, \ldots, \mathbf{x}^{\left(N_{s p}\right)}$

\section{Radial Basis Function Overview}

Radial basis function (RBF) interpolation is similar to kriging in the sense that they are based on Gaussian correlation functions. However, in this paper RBF interpolation refers to an approximation method based on Gaussian correlation functions that does not include a constant global approximation term, unlike kriging. The method of RBF interpolation used in this study is based on the method employed in Ref. 13. A brief description of the methodology for generating the RBF surrogate is described next.

In RBF surrogates, the approximate response is a weighted sum of basis functions:

$$
\hat{y}=\sum_{i=1}^{N_{s p}} w_{i} \phi_{\mathrm{RBF}}\left(\left\|\mathbf{x}-\mathbf{x}^{(i)}\right\|\right)
$$


where $\phi_{\mathrm{RBF}}(*)$ is typically a non-linear function depending on the Euclidean distance (denoted by $\left\|\mathbf{x}-\mathbf{x}^{(i)}\right\|$ ) between two design points. The coefficients, $w_{i}$, must be found such that the surrogate interpolates the initial data points. Thus, the following condition must be satisfied for $j=1, \ldots, N_{s p}$ :

$$
\hat{y}\left(\mathbf{x}^{(j)}\right)=\sum_{i=1}^{N_{s p}} w_{i} \phi_{\mathrm{RBF}}\left(\left\|\mathbf{x}^{(j)}-\mathbf{x}^{(i)}\right\|\right)
$$

By defining the vectors $\mathbf{w}=\left[w_{1}, w_{2}, \ldots, w_{N_{s p}}\right]^{\mathrm{T}}, \mathbf{y}=\left[y_{1}, y_{2}, \ldots, y_{N_{s p}}\right]$, and the $N_{s p} \times N_{s p}$ matrix $\mathbf{\Phi}_{\mathrm{RBF}}$ with elements $\left(\Phi_{\mathrm{RBF}}\right)_{i j}=\phi_{\mathrm{RBF}}\left(\left|\mathbf{x}^{(i)}-\mathbf{x}^{(j)}\right|\right)$, Eq. (21) can be rewritten as

$$
\boldsymbol{\Phi}_{\mathrm{RBF}} \mathbf{w}=\mathbf{y}^{\mathrm{T}}
$$

If the inverse of $\boldsymbol{\Phi}_{\mathrm{RBF}}$ exists, then the weighting coefficients are

$$
\mathbf{w}=\left(\boldsymbol{\Phi}_{\mathrm{RBF}}\right)^{-1} \mathbf{y}^{\mathrm{T}}
$$

and the RBF surrogate is

$$
\hat{y}_{R B F}(\mathbf{x})=\phi_{\mathrm{RBF}} \mathbf{w}=\phi_{\mathrm{RBF}}\left(\boldsymbol{\Phi}_{\mathrm{RBF}}\right)^{-1} \mathbf{y}^{\mathrm{T}}
$$

where

$$
\phi_{\mathrm{RBF}}=\left[\phi_{\mathrm{RBF}}\left(\left\|\mathbf{x}-\mathbf{x}^{(1)}\right\|\right), \phi_{\mathrm{RBF}}\left(\left\|\mathbf{x}-\mathbf{x}^{(2)}\right\|\right), \ldots, \phi_{\mathrm{RBF}}\left(\left\|\mathbf{x}-\mathbf{x}^{\left(N_{s p}\right)}\right\|\right)\right] .
$$

As stated above, Eq. (24) shows that the RBF surrogate does not include a constant global approximation term, unlike Eq. (18) which includes $\hat{\beta}$. Gaussian correlation functions of the form given by Eq. 26 are used for the basis functions in Eq. 20.

$$
\phi_{\mathrm{RBF}}(\eta)=\exp \left(-\eta^{2} / 2 \tau^{2}\right)
$$

In this case, the dummy variable $\eta$ would be $\left\|\mathbf{x}-\mathbf{x}^{(i)}\right\|$. As described in Ref. 13, the fitting parameter $\tau$ is found by leave-one-out cross validation. This process is described below:

1. The design variables are scaled to vary from 0 to 1 . The possible values of $\tau$ are then spread over the domain $\left[10^{-2}, 10^{1}\right]$ on a logarithmic scale, which is an appropriate domain when the design variables are scaled as mentioned. ${ }^{13}$

2. For each value of $\tau, N_{s p}$ RBF models are created, leaving one interpolation point out each time, as if only $N_{s p}-1$ interpolation points exist. Therefore, for each value of $\tau, N_{s p}$ evaluations of Eq. 24 are required and each evaluation involves the inversion of the $N_{s p} \times N_{s p}$ matrix $\boldsymbol{\Phi}_{\mathrm{RBF}}$. Since a large set of $\tau$ can lead to an excessive number of evaluations of Eq. 24, only 15 values for $\tau$ were considered so that the RBF remains computationally tractable.

3. The difference between the true response at the left out point and the response predicted at the left out point by the RBF model based on $N_{s p}-1$ points is computed.

4. The value of $\tau$ that minimizes the sum of these residuals is selected as the fitting parameter.

\section{Results}

This section presents the accuracies of the approximation methods that have been described together with vibration reduction results using surrogate objective functions. The helicopter configuration used in all computations is given in Table 1. The simulations are conducted at an advance ratio of 0.15 and descent angle of $6^{\circ}$, where high vibration levels due to strong blade vortex interaction (BVI) are encountered..$^{38}$ Figure 6 illustrates a helicopter in descent; this figure is also employed for the propulsive trim calculation.

In addition to the information provided in Table 1, additional information is needed on the baseline design, the objective function, the constraints, and specific locations of the spanwise stations where the design variables are specified. This information is provided below.

The baseline design was determined by tuning $x_{1}, x_{2}, t_{1}, t_{2}, t_{3}$ and $m_{\text {filler }}$ such that the baseline blade has fundamental frequencies similar to an MBB BO-105 blade, while assuming aluminum material properties. The baseline blade was assumed to have uniform spanwise properties, and the non-structural mass at the 
Table 1. Rotor and helicopter parameters needed for the computations.

\begin{tabular}{ll}
\multicolumn{1}{l}{ Dimensional Data } & \\
$R=4.91 \mathrm{~m}$ & $\Omega=425 \mathrm{RPM}$ \\
Non-Dimensional Data & \\
$N_{b}=4$ & $c=0.05498 R$ \\
$\beta_{p}=0.0^{\circ}$ & $C_{d o}=0.01$ \\
$\theta_{\mathrm{pt}}=0^{\circ}$ & $\alpha_{\mathrm{d}}=6^{\circ}$ \\
$\mu=0.15$ & $C_{W}=0.005$ \\
$\sigma=0.07$ & $C_{d f}=0.01$ \\
$X_{F A}=0.0$ & $Z_{F A}=0.3$ \\
$X_{F C}=0.0$ & $Z_{F C}=0.3$ \\
& \\
\hline
\end{tabular}

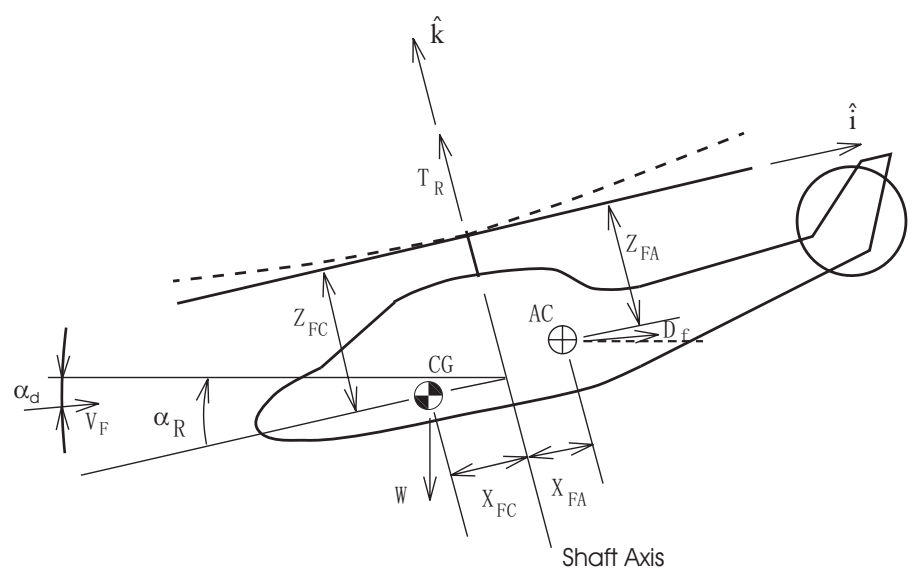

Figure 6. Helicopter in descent flight condition.

Table 2. Fixed parameters defining the structure and root section.

$$
\begin{aligned}
& \text { Aluminum Material Properties } \\
& E=70.7 \mathrm{GPa} \\
& \nu=0.33 \\
& \rho_{\text {struct }}=2700 \mathrm{~kg} / \mathrm{m}^{3}
\end{aligned}
$$

Non-structural Filler Mass Density

$\rho_{\text {filler }}=237.4 \mathrm{~kg} / \mathrm{m}^{3}$

Baseline Cross-Sectional Parameters

\begin{tabular}{ll}
$x_{1}=65.4 \mathrm{~mm}$ & $x_{2}=111.6 \mathrm{~mm}$ \\
$t_{1}=4.66 \mathrm{~mm}$ & $t_{2}=8.48 \mathrm{~mm}$ \\
$t_{3}=2.21 \mathrm{~mm}$ & $m_{n s}=0$ \\
\hline
\end{tabular}


Table 3. Comparison of baseline fundamental frequencies to MBB BO-105 values.

\begin{tabular}{l|c|c|c} 
Frequency & MBB BO-105 & Baseline Blade & \% Difference \\
\hline$\omega_{F 1}$ & $1.125 / \mathrm{rev}$ & $1.080 / \mathrm{rev}$ & $-4.0 \%$ \\
\hline$\omega_{L 1}$ & $0.732 / \mathrm{rev}$ & $0.729 / \mathrm{rev}$ & $-0.4 \%$ \\
\hline$\omega_{T 1}$ & $3.176 / \mathrm{rev}$ & $3.173 / \mathrm{rev}$ & $-0.09 \%$ \\
\hline
\end{tabular}

elastic axis was assumed to be zero. The material properties and the baseline cross-sectional design are given in Table 2, and a comparison of the baseline fundamental frequencies with the MBB BO-105 fundamental frequencies is given in Table 3. It is not surprising that all three frequencies could not be fit exactly because the MBB BO-105 blade is a composite blade, while the current study is based on an isotropic blade model.

The weighting factors in the objective function, $K_{S}$ and $K_{M}$, are selected to be 1 . These weighting factors result in an objective function which represents the sum of the 4/rev oscillatory hub shear resultant and the $4 / \mathrm{rev}$ oscillatory hub moment resultant in the hub-fixed non-rotating frame. For this study, the following side constraints are enforced:

$$
\begin{gathered}
1.0 \mathrm{~mm} \leq t_{1} \leq 8.0 \mathrm{~mm} \\
1.0 \mathrm{~mm} \leq t_{2}, t_{3} \leq 12.0 \mathrm{~mm} \\
0.0 \leq m_{n s} / m_{0} \leq 0.25
\end{gathered}
$$

The upper and lower bounds used for the frequency placement constraints are given in Table 4, and are similar to those used in Ref. 39, which also used cross-sectional dimensions as design variables.

Table 4. Upper and lower bounds on the fundamental frequencies (/rev).

\begin{tabular}{c|ccc} 
& Flap & Lag & Torsion \\
\hline$\omega_{U}$ & 1.20 & 0.80 & 6.50 \\
$\omega_{L}$ & 1.05 & 0.60 & 2.50
\end{tabular}

In the aeroelastic stability constraints given by Eq. 7, the minimum acceptable damping for all modes, $\left(\zeta_{k}\right)_{\min }$, is chosen to be 0.01 , as in Ref. 8. Additionally, the constraints are modified for the $2^{\text {nd }}$ lag mode, which can sometimes be slightly unstable. To prevent this situation, a small amount of structural damping is added to this mode, as in Ref. 2. For this study, $0.5 \%$ structural damping is added to stabilize the $2^{\text {nd }}$ lag mode of the baseline blade.

The rotor blade was discretized into the 6 finite elements shown in Fig. 7 and the design variables were defined at two nodal locations - the $68 \%$ and tip stations - resulting in a total of 8 design variables. These two blade stations were chosen because previous studies have shown that (a)non-structural masses are most effective for vibration reduction when they are distributed over the outboard $1 / 3$ of the blade ${ }^{40,41}$ and (b) a similar configuration has been used successfully for vibration reduction in Ref. 27 . The root cross-sectional variables were fixed at the baseline values and the cross-sectional variables were assumed to vary linearly between stations. The non-structural mass at the elastic axis inboard of the $68 \%$ station was set to zero.

\section{A. Surrogate Accuracy Results}

In order to test the predictive capability of the approximation methods discussed in Section IV the surrogates for the hub shears and moments (see Eq. 8) were used to predict vibratory loads at 45 design points spread throughout the design space. Four surrogate methods were compared, including $2_{n d}$ order polynomials, $\mathrm{RBF}$, ordinary kriging where $p$ is fixed at 2 , and kriging where $p$ is not fixed. The predicted vibratory loads from the surrogates were then compared to the "actual" vibratory loads, which were obtained by conducting helicopter simulations at 45 test points. The error measures used to quantify accuracy were average percent 


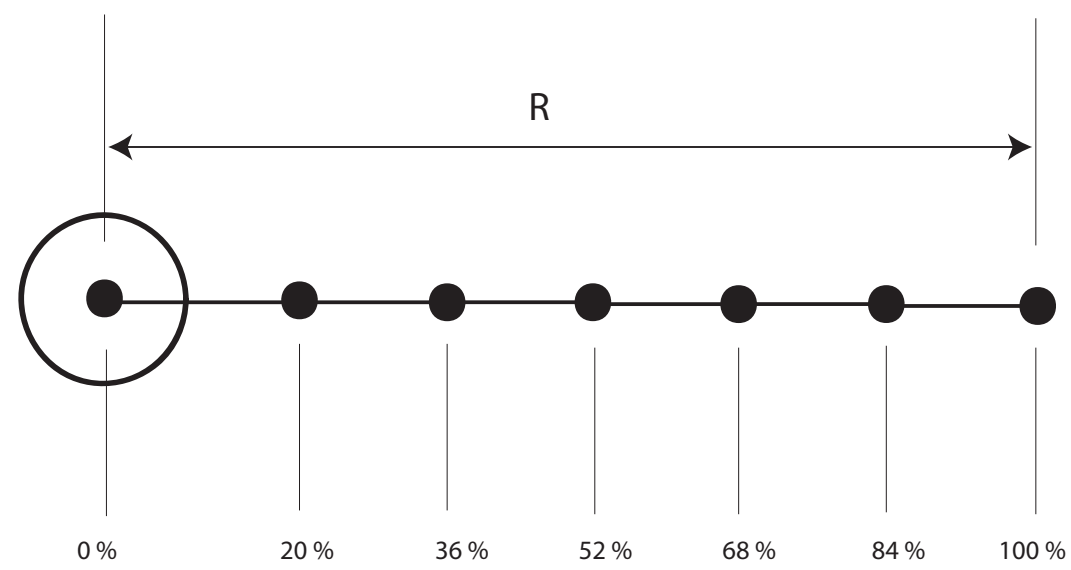

Figure 7. Finite element discretization of the blade.

error, maximum percent error, and minimum percent error. The absolute percent error is simply

$$
\varepsilon_{i}=\frac{\left|y^{(i)}-\hat{y}^{(i)}\right|}{y^{(i)}}
$$

where $y^{(i)}$ is the "actual" response computed by the helicopter simulation for a blade design defined by $\mathbf{x}^{(i)}$ and $\hat{y}^{(i)}$ is the response predicted by the surrogate at $\mathbf{x}^{(i)}$. The average percent error, maximum percent error, and minimum percent error are:

$$
\begin{aligned}
\varepsilon_{a v g} & =\frac{\sum_{i=1}^{N_{t p}} \varepsilon_{i}}{N_{t p}} \\
\varepsilon_{\max } & =\operatorname{Max}\left\{\varepsilon_{1}, \ldots, \varepsilon_{N_{t p}}\right\} \\
\varepsilon_{\text {min }} & =\operatorname{Min}\left\{\varepsilon_{1}, \ldots, \varepsilon_{N_{t p}}\right\}
\end{aligned}
$$

where $N_{t p}$ is the number of test points. The minimum and maximum percent errors represent the best and worse predictive errors respectively. These error measures are localized since they only represent one point of the 45 test points. However, the average percent error is a more global representation of a surrogate's predictive capability since all 45 test points are included.

Two optimal Latin hypercubes(OLH's) were used to create the fitting points for the surrogates - an 80 point OLH and a 250 point OLH. Since the computational cost of evaluating the actual constraints is low, the constraints at each point in the OLH were evaluated first. Designs that violated the constraints were removed from the data set so that expensive forward flight simulations would not be wasted. From the 80 point OLH, 61 points were feasible and had converged trim solutions; while out of the 250 point OLH, 151 points were feasible with converged trim solutions.

One of the advantages of surrogate based optimization with design of computer experiments is that each simulation corresponding to a design point in the OLH can be run independently of the other design points, and therefore the simulations can be run in parallel. The helicopter simulations were run on a parallel cluster with $1.8 \mathrm{Ghz}$ Opteron processors. The simulation time was about 4 hours per simulation and 10 to 20 simulations were run simultaneously. Results for the error measures for each surrogate are given in Figs. 8a- 8c for the 61 point design of computer experiment, and Figs. 9a- 9c for the 151 point design of computer experiment.

High maximum fitting errors, particularly for the horizontal and vertical hub shear components $F_{4 X}$ and $F_{4 Z}$, can be seen in Figure $8 \mathrm{a}$ for the 61 point data set with all four surrogate approaches. This suggests that there are localized regions in the design space where the surrogates may not be reliable. Although the $2^{\text {nd }}$ order polynomials are clearly the least accurate, none of the surrogates distinguish themselves as being the best approximation method in terms of maximum and minimum percent errors. Figure $8 \mathrm{c}$ shows that the kriging models are superior in terms of average errors, which range from 10-55\% for the kriging models. The results for average error shows that both kriging models are superior in terms of accurately modeling 

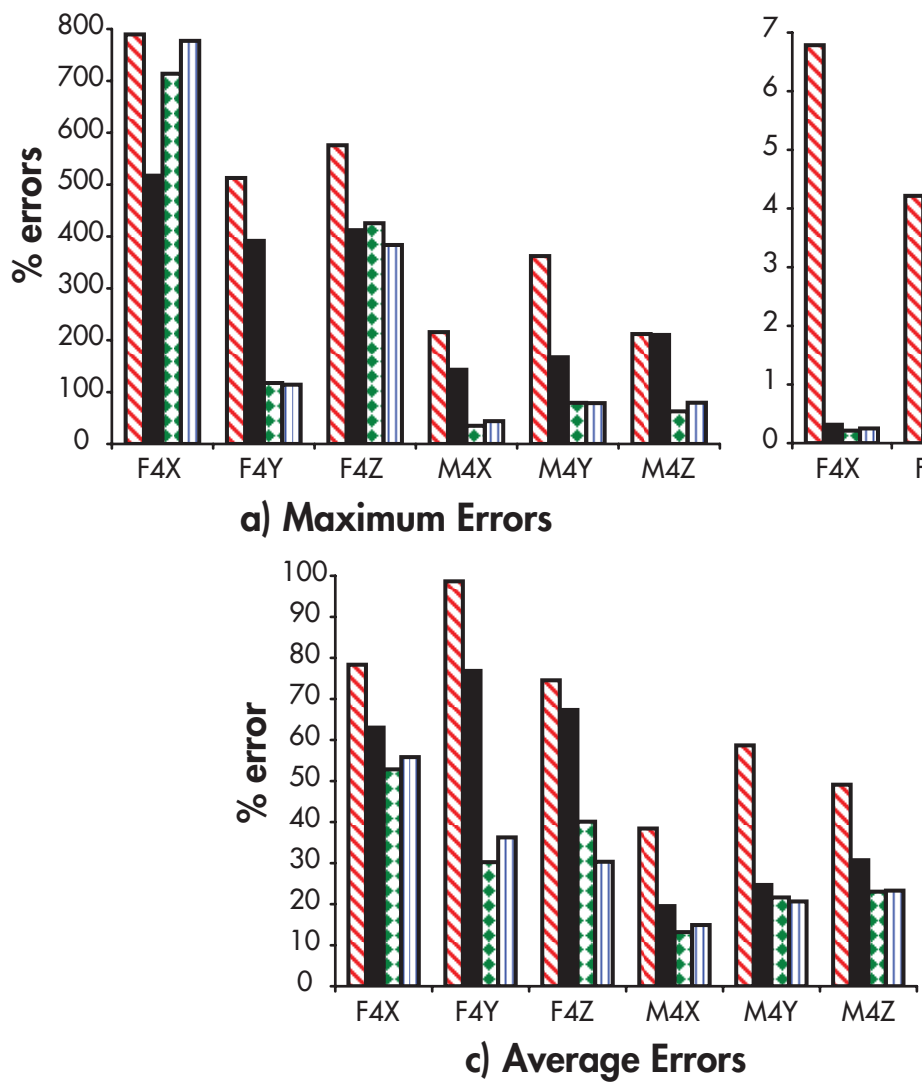
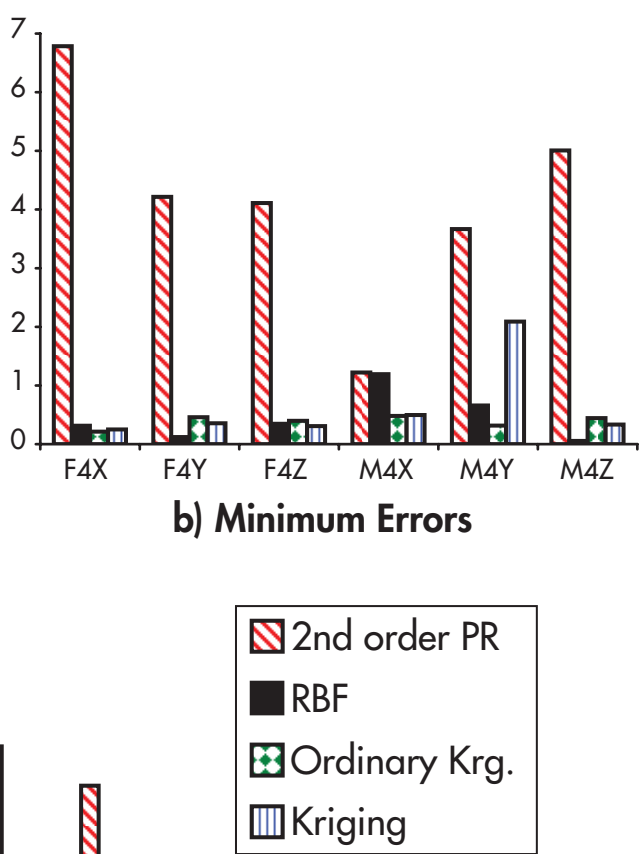

Figure 8. Surrogate errors in percentage for the 61 point data set.

vibrations over the entire design space. By comparison, the two kriging models seem to provide predictions with similar degrees of accuracy.

When 151 data points are used, all three error measures for each surrogate are reduced compared to the 61 point errors. However, Figure 9a shows that all of the surrogates still produce poor predictions in certain localized regions of the design space, although they are noticeably better compared to Fig. 8a (Note the difference in scales on the vertical axes between Fig. 9 and Fig. 8). Similar to the 61 point case observed earlier, Figure $9 \mathrm{c}$ shows that both kriging models are superior at approximating the vibratory loads over the entire design space, with average errors of $5-37 \%$. In addition, it is not evident that one kriging model is superior to the other when using 151 interpolation points. Overall, it can be concluded from Figures 8- 9 that $2^{\text {nd }}$ order polynomials are the worst of the surrogate methods and kriging is the best method for capturing the behavior of the vibratory loads over the entire design space. Furthermore, there is no clear advantage in fitting accuracy in the more general kriging method, i.e. where $p$ is not fixed at 2, compared to ordinary kriging.

\section{B. Optimization Results}

The surrogate based optimization (SBO) conducted in this study is non-adaptive, otherwise known as the "one-shot" approach, which means that the surrogate is not updated with objective function evaluations as the optimization progresses. While it is important to use an adaptive method so that the optimization process does not lead to regions of high uncertainty in the surrogate, the focus of this study was on the effectiveness of the approximation techniques and not on the search algorithm. The optimization of the surrogate objective function was conducted with the iSIGHT ${ }^{42}$ software package using the Multi-Island Genetic Algorithm for a global search of the surrogate, followed by a local gradient based search. Only the surrogates based on 151 data points were used for optimization since they were the most accurate. In addition, optimization results based on kriging when $p$ is not fixed at 2 are not shown because they were identical to the ordinary kriging optimization results. 

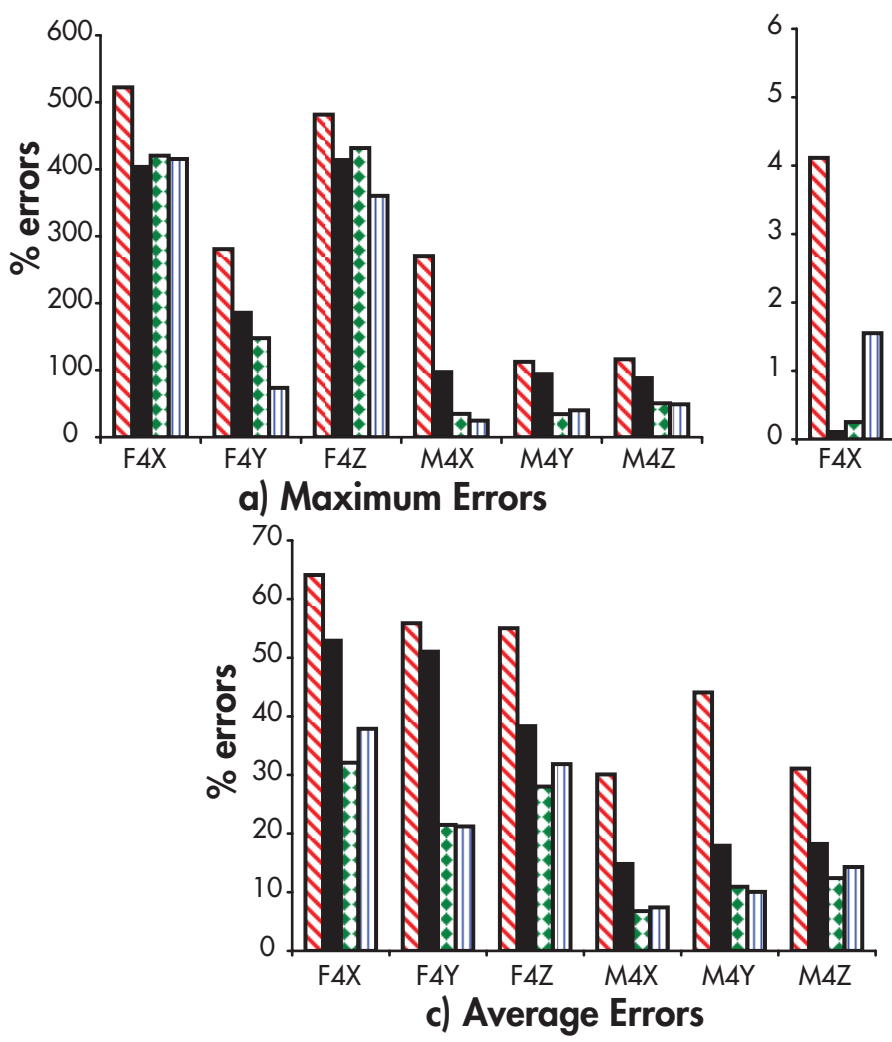
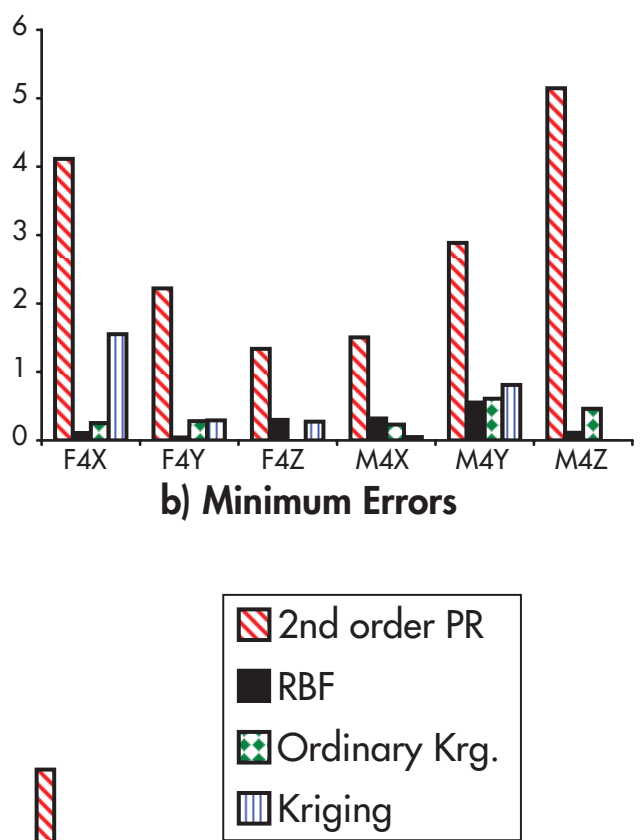

Figure 9. Surrogate errors in percentage for the 151 point data set.

In Table 5, the predicted vibration reductions at the SBO optimal designs are compared with the "actual" vibration reductions computed by the helicopter simulation at the SBO optimal designs. Note that vibration reductions are computed relative to the vibration levels of the baseline blade, and each surrogate generates a different optimal design. The results in Table 5 clearly show that the kriging model leads to the most

Table 5. Comparison of predicted vibration reductions vs "actual" vibration reductions.

\begin{tabular}{l|c|c} 
Surrogate & $\begin{array}{c}\text { Predicted \% Vibration Reduction } \\
\text { (relative to baseline) }\end{array}$ & $\begin{array}{c}\text { Actual \% Vibration Reduction } \\
\text { (relative to baseline) }\end{array}$ \\
\hline 2nd order poly. & $42.84 \%$ & $41.77 \%$ \\
\hline RBF & $79.31 \%$ & $74.03 \%$ \\
\hline Ordinary krg. & $84.27 \%$ & $83.71 \%$ \\
\hline
\end{tabular}

optimal design. Furthermore, the $2^{\text {nd }}$ order polynomial is inferior compared to the kriging and RBF methods. The significant levels of vibration reduction shown in Table 5 obtained using the RBF and ordinary kriging surrogates suggest that the baseline blade may not be a good design in terms of vibrations. The choice of a baseline blade is revisited later in this section. The results in Table 5 are deceiving since they may lead one to believe that the surrogates are extremely accurate at the optimal designs, because the vibration reductions predicted by the surrogates are close to the actual vibration reductions computed by the helicopter simulations. However, the surrogates were not fit to the vibration objective function directly; rather they were fit to the individual hub shears and moments which form the objective function (see Eq. 8). Therefore, a more appropriate measures of the surrogate accuracies at the optimal designs are obtained by comparing the approximated hub shears and moments to the actual hub shears and moments computed by the helicopter simulation. This comparison is shown in Fig. 10, which shows that surrogate predictions at their respective optimal designs are not as accurate as Table 5 suggests. In fact, for $\hat{F}_{4 X}$ and $\hat{F}_{4 Y}$ the $2^{\text {nd }}$ order polynomial 
predicts negative values, which also occurs for the RBF prediction of $\hat{M}_{4 Z}$ (Note that the negative values are not reflected in Fig. 10 since absolute errors are plotted). Negative values are not possible since the quantities being modeled represent the amplitudes of the 4/rev hub loads. Therefore it is advisable to model the sine and cosine components of the oscillatory hub loads and then compute the amplitudes based on the surrogate components in order to guarantee positive amplitudes. Although Figure 10 shows that the surrogates at the optimal designs are not as accurate as Table 5 suggests, the amount of vibration reduction shown in Table 5 demonstrates that enough of the behavior of the objective function has been captured such that the surrogates predict regions of improved design.

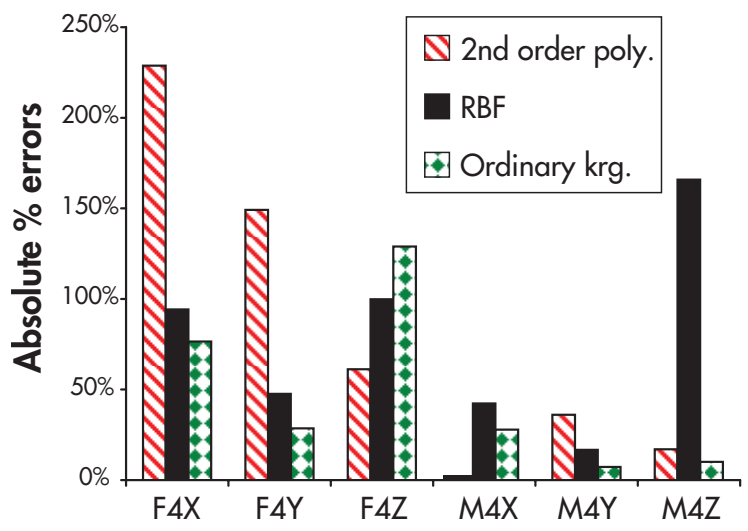

Figure 10. Absolute \% errors of the hub loads predicted by the surrogates relative to the actual hub loads at the optimal designs.

Figure 11 shows the optimal hub loads computed by the helicopter simulation at the optimum design from each surrogate, along with the baseline hub loads. From this figure, it is clear that all surrogates effectively reduce all of the vibratory load components. Moreover, ordinary kriging results in the lowest values of each hub shear and moment, except $M_{4 Y}$ where the RBF surrogate is only slightly better, which further supports the argument that ordinary kriging is the most effective surrogate for producing reduced vibration designs.

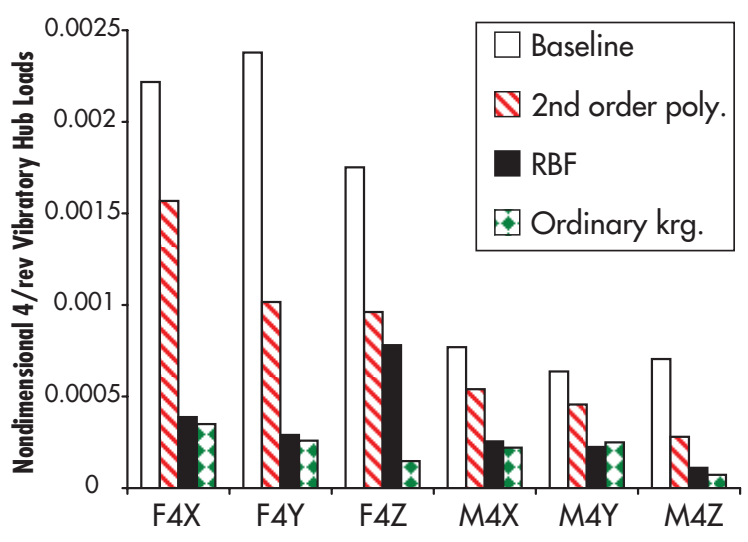

Figure 11. Comparison of optimal vibratory loads with baseline values

The optimal and baseline designs summarized in Table 6 show that the RBF and kriging optimal designs are similar in all the design variables, except $t_{3}$ at both the $68 \%$ and tip stations. This suggests that the optimal designs obtained by RBF and kriging surrogates fall in a general region of the design space with reduced vibrations, and that the kriging method is capable of identifying a better solution within the region.

As mentioned earlier, the baseline blade seems to have poor vibration characteristics. Therefore a more accurate representation of an MBB BO-105 blade, which has better vibration characteristics than the baseline blade, is used for comparison against the optimal hub loads. The more accurate MBB BO-105 blade design reproduces all of the fundamental frequencies given in Table 3 exactly, which could not be done with the baseline design in this study. The cross-sectional properties of the blade resembling an MBB BO-105 were 
Table 6. Optimal and baseline designs.

\begin{tabular}{c|c|c|c|c} 
Design Variable & Baseline & 2nd order poly. & RBF & Ordinary krg. \\
\hline$t_{1} @ 68 \% R(\mathrm{~mm})$ & 4.657 & 3.029 & 1.568 & 1.971 \\
\hline$t_{2} @ 68 \% R(\mathrm{~mm})$ & 8.482 & 5.717 & 2.916 & 2.709 \\
\hline$t_{3} @ 68 \% R(\mathrm{~mm})$ & 2.214 & 5.161 & 5.165 & 3.342 \\
\hline$m_{n s} / m_{0} @ 68 \% R$ & none & 0.135 & 0.242 & 0.222 \\
\hline$t_{1} @ 100 \% R(\mathrm{~mm})$ & 4.657 & 5.158 & 6.739 & 7.035 \\
\hline$t_{2} @ 100 \% R(\mathrm{~mm})$ & 8.482 & 7.050 & 10.500 & 9.235 \\
\hline$t_{3} @ 100 \% R(\mathrm{~mm})$ & 2.214 & 5.465 & 3.218 & 7.773 \\
\hline$m_{n s} / m_{0} @ 100 \% R$ & none & 0.172 & 0.246 & 0.239 \\
\hline
\end{tabular}

input directly into the helicopter simulation rather than calculate them from cross-sectional dimensions and material properties. Table 7 compares the actual levels of vibration reduction obtained from the helicopter simulation using the optimal blade designs of Table 6 with the vibration levels of the blade resembling an MBB BO-105 blade. Even relative to the better blade design, the RBF and ordinary kriging surrogates still produce significant levels of vibration reduction. By contrast, the optimal design produced by the 2nd order polynomial is worse than the MBB BO-105 blade.

Table 7. Comparison of actual \% vibration reductions relative to the MBB BO-105 values.

\begin{tabular}{l|c} 
Surrogate & Vibration Reduction \\
\hline 2nd order poly. & $17.39 \%$ increase \\
\hline RBF & $47.65 \%$ \\
\hline Ordinary krg. & $67.16 \%$ \\
\hline
\end{tabular}

The 4/rev vibratory hub loads are shown in Fig. 12 for the optimal designs and the blade resembling an MBB BO-105. All of the optimal designs have significantly reduced the dominant vertical shear component, though all the other vibratory components are somewhat increased. However, these components are not substantially increased by the optimum designs, with the exception of the $2^{\text {nd }}$ order polynomial case.

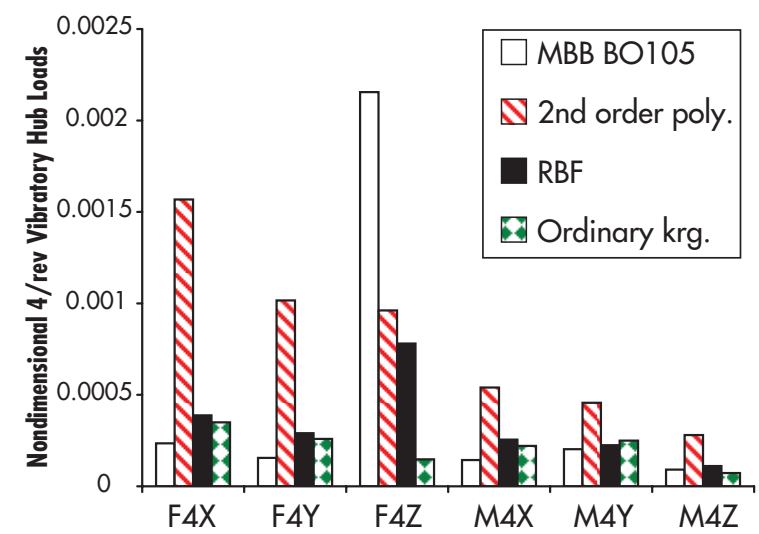

Figure 12. Hub loads for the optimal designs, and the MBB BO-105 blade. 


\section{Conclusions}

The results in this paper demonstrate that stochastic process based approximation methods like kriging and RBF's can lead to blade designs with reduced vibration levels. Even with localized regions in the design space where the prediction based on the surrogates is poor, they still captured enough of the behavior over the entire design space such that they could be used to find regions of improved design. The principal results from this study are summarized below.

1. Among the methods considered, kriging was the most effective method for approximating vibratory loads over the entire design space, and for locating an optimum blade design. Although the average accuracies of kriging were the best, the surrogate was susceptible to extremely high errors at certain design points. The $2^{\text {nd }}$ order polynomial was the worse approximation method considered.

2. Allowing the parameters $p$ to vary in the kriging fitting process does not offer any clear advantages over ordinary kriging.

3. Using ordinary kriging and RBF surrogates, vibration reductions relative to an MBB BO-105 blade model of $67 \%$ and $48 \%$, respectively, could be achieved.

4. The high maximum errors suggest that kriging and RBF surrogates cannot be used for precise predictions of vibrations everywhere in the design space (at least without adding more interpolation points), although they are still useful in finding optimal designs.

\section{Appendix A}

The development of the perturbation equations used in the linearized stability analysis are given in this appendix. The process used in Ref. 8 has been modified to take into account the RFA aerodynamic model. In Ref. 8, the blade equations of motion were only a function of the blade response and trim parameters, i.e.,

$$
\mathbf{f}_{b}\left(\mathbf{q}_{b}, \dot{\mathbf{q}}_{b}, \ddot{\mathbf{q}}_{b}, \mathbf{q}_{\mathbf{t}}\right)=\mathbf{0}
$$

where $\mathbf{q}_{b}$ is the vector of generalized modal coordinates representing the blade degrees of freedom and $\mathbf{q}_{t}$ is the vector of trim parameters which are uniform inflow and collective pitch for hover. In this study, the blade equations of motion are written as

$$
\mathbf{f}_{b}\left(\mathbf{q}_{b}, \dot{\mathbf{q}}_{b}, \ddot{\mathbf{q}}_{b}, \mathbf{x}_{a}, \mathbf{q}_{t}\right)=\mathbf{0}
$$

where $\mathbf{x}_{a}$ is the vector of aerodynamic states introduced by the RFA aerodynamic model. Correspondingly, there is a set of governing ODE's for the aerodynamic state vector,

$$
\dot{\mathbf{x}}_{a}=\mathbf{g}_{a}\left(\mathbf{q}_{b}, \dot{\mathbf{q}}_{b}, \ddot{\mathbf{q}}_{b}, \mathbf{x}_{a}, \mathbf{q}_{\mathbf{t}}\right) .
$$

Equations 35 and 36 represent the coupled set of ordinary differential equations that govern the rotor blade system. The components of Eqs. 35 and Eqs. 36 are formed numerically as part of the helicopter simulation, and detailed equations for the components can be found in Refs. 22 and 43. Since these equations are coupled, the combined system must be linearized. The linearization process is now discussed.

Following the development in Ref. 22, Eq. 35 is rewritten as

$$
\mathbf{f}_{b}=\mathbf{g}_{b}\left(\mathbf{q}_{b}, \dot{\mathbf{q}}_{b}, \mathbf{x}_{a}, \mathbf{q}_{\mathbf{t}}\right)+\mathbf{M}\left(\mathbf{q}_{b}, \mathbf{q}_{\mathbf{t}}\right) \ddot{\mathbf{q}}_{b}=\mathbf{0}
$$

where

$$
\mathbf{M} \equiv \frac{\partial \mathbf{f}_{b}}{\partial \ddot{\mathbf{q}}_{b}} .
$$

Dependence on $\ddot{\mathbf{q}}_{b}$ is eliminated by substituting Eq.( 38 ) into Eq.( 36), yielding

$$
\dot{\mathbf{x}}_{a}=\mathbf{g}_{a R}\left(\mathbf{q}_{b}, \dot{\mathbf{q}}_{b}, \mathbf{x}_{a}, \mathbf{q}_{\mathbf{t}}\right) .
$$

Perturbing Eq.( 35$)$ about the static equilibrium and neglecting higher order terms gives

$$
\left[\frac{\partial \mathbf{f}_{b}}{\partial \ddot{\mathbf{q}}_{b}}\right]_{\mathbf{y}_{0}} \Delta \ddot{\mathbf{q}}_{b}+\left[\frac{\partial \mathbf{f}_{b}}{\partial \dot{\mathbf{q}}_{b}}\right]_{\mathbf{y}_{0}} \Delta \dot{\mathbf{q}}_{b}+\left[\frac{\partial \mathbf{f}_{b}}{\partial \mathbf{q}_{b}}\right]_{\mathbf{y}_{0}} \Delta \mathbf{q}_{b}+\left[\frac{\partial \mathbf{f}_{b}}{\partial \mathbf{x}_{a}}\right]_{\mathbf{y}_{0}} \Delta \mathbf{x}_{a}=\mathbf{0}
$$


where $\mathbf{y}_{0}$ is the static equilibrium vector and is given by

$$
\mathbf{y}_{0}=\left[\begin{array}{c}
\mathbf{q}_{b 0} \\
\dot{\mathbf{q}}_{b 0} \\
\dot{\mathbf{x}}_{a 0}
\end{array}\right]
$$

The "0" subscript denotes static equilibrium solution.

From Eq. 37,

$$
\begin{gathered}
{\left[\frac{\partial \mathbf{f}_{b}}{\partial \dot{\mathbf{q}}_{b}}\right]_{\mathbf{y}_{0}}=\left[\frac{\partial \mathbf{g}_{b}}{\partial \dot{\mathbf{q}}_{b}}\right]_{\mathbf{y}_{0}}} \\
{\left[\frac{\partial \mathbf{f}_{b}}{\partial \mathbf{q}_{b}}\right]_{\mathbf{y}_{0}}=\left[\frac{\partial \mathbf{g}_{b}}{\partial \mathbf{q}_{b}}\right]_{\mathbf{y}_{0}}} \\
{\left[\frac{\partial \mathbf{f}_{b}}{\partial \mathbf{x}_{a}}\right]_{\mathbf{y}_{0}}=\left[\frac{\partial \mathbf{g}_{b}}{\partial \mathbf{x}_{a}}\right]_{\mathbf{y}_{0}} .}
\end{gathered}
$$

Substituting Eqs.( 42- 44) and Eq.( 38 ) into Eq.( 40) gives

$$
[\mathbf{M}]_{\mathbf{y}_{0}} \Delta \ddot{\mathbf{q}}_{b}+\left[\frac{\partial \mathbf{g}_{b}}{\partial \dot{\mathbf{q}}_{b}}\right]_{\mathbf{y}_{0}} \Delta \dot{\mathbf{q}}_{b}+\left[\frac{\partial \mathbf{g}_{b}}{\partial \mathbf{q}_{b}}\right]_{\mathbf{y}_{0}} \Delta \mathbf{q}_{b}+\left[\frac{\partial \mathbf{g}_{b}}{\partial \mathbf{x}_{a}}\right]_{\mathbf{y}_{0}} \Delta \mathbf{x}_{a}=\mathbf{0}
$$

Solving for $\Delta \ddot{\mathbf{q}}_{b}$ yields

$$
\Delta \ddot{\mathbf{q}}_{b}=-\mathbf{M}^{-1}\left[\frac{\partial \mathbf{g}_{b}}{\partial \dot{\mathbf{q}}_{b}}\right]_{\mathbf{y}_{0}} \Delta \dot{\mathbf{q}}_{b}-\mathbf{M}^{-1}\left[\frac{\partial \mathbf{g}_{b}}{\partial \mathbf{q}_{b}}\right]_{\mathbf{y}_{0}} \Delta \mathbf{q}_{b}-\mathbf{M}^{-1}\left[\frac{\partial \mathbf{g}_{b}}{\partial \mathbf{x}_{a}}\right]_{\mathbf{y}_{0}} \Delta \mathbf{x}_{a} .
$$

Similarly, Eq. 39 can be linearized, yielding

$$
\Delta \dot{\mathbf{x}}_{a}=\left[\frac{\partial \mathbf{g}_{a R}}{\partial \dot{\mathbf{q}}_{b}}\right]_{\mathbf{y}_{0}} \Delta \dot{\mathbf{q}}_{b}+\left[\frac{\partial \mathbf{g}_{a R}}{\partial \mathbf{q}_{b}}\right]_{\mathbf{y}_{0}} \Delta \mathbf{q}_{b}+\left[\frac{\partial \mathbf{g}_{a R}}{\partial \mathbf{x}_{a}}\right]_{\mathbf{y}_{0}} \Delta \mathbf{x}_{a} .
$$

Combining Eqs. ( 46) and ( 47) with the trivial perturbation equation $\Delta \dot{\mathbf{y}}_{b}=\Delta \dot{\mathbf{y}}_{b}$ into first-order state space form gives

$$
\dot{\mathbf{z}}=\left[\mathbf{A}\left(\mathbf{y}_{0}\right)\right] \mathbf{z}
$$

where

$$
\left[\mathbf{A}\left(\mathbf{y}_{0}\right)\right]=\left[\begin{array}{ccc}
\mathbf{0} & \mathbf{I} & \mathbf{0} \\
-\mathbf{M}^{-1}\left[\frac{\partial \mathbf{g}_{b}}{\partial \mathbf{q}_{b}}\right]_{\mathbf{y}_{0}} & -\mathbf{M}^{-1}\left[\frac{\partial \mathbf{g}_{b}}{\partial \dot{\mathbf{q}}_{b}}\right]_{\mathbf{y}_{0}} & -\mathbf{M}^{-1}\left[\frac{\partial \mathbf{g}_{b}}{\partial \mathbf{x}_{a}}\right]_{\mathbf{y}_{0}} \\
{\left[\frac{\partial \mathbf{g}_{a R}}{\partial \mathbf{q}_{b}}\right]_{\mathbf{y}_{0}}} & {\left[\frac{\partial \mathbf{g}_{a R}}{\partial \dot{\mathbf{q}}_{b}}\right]_{\mathbf{y}_{0}}} & {\left[\frac{\partial \mathbf{g}_{a R}}{\partial \mathbf{x}_{a}}\right]_{\mathbf{y}_{0}}}
\end{array}\right]
$$

and

$$
\mathbf{z} \equiv \Delta \mathbf{y}=\left[\begin{array}{c}
\Delta \mathbf{q}_{b} \\
\Delta \dot{\mathbf{q}}_{b} \\
\Delta \mathbf{x}_{a}
\end{array}\right]
$$

As mentioned, the stability of the system is determined by the eigenvalues of $\mathbf{A}$.

\section{Acknowledgments}

This research was supported in part by the FXB Center for Rotary and Fixed Wing Air Vehicle Design, and by ARO grant 02-1-0202 with Dr. B. LaMattina as grant monitor. The authors are grateful to Professor Raphael Haftka for his valuable advice, and Professor Panos Papalambros and Dr. Subroto Gunawan for providing the kriging computer algorithms which were used in this study. 


\section{References}

${ }^{1}$ Friedmann, P., "Helicopter Vibration Reduction Using Structural Optimization with Aeroelastic/Multidisciplinary Constraints - A Survey," Journal of Aircraft, Vol. 28, No. 1, January 1991, pp. 8-21.

${ }^{2}$ Yuan, K. and Friedmann, P., "Structural Optimization for Vibratory Loads Reduction of Composite Helicopter Rotor Blades with Advanced Geometry Tips," Journal of the American Helicopter Society, Vol. 43, No. 3, July 1998, pp. $246-256$.

${ }^{3}$ Celi, R., "Recent Applications of Design Optimization to Rotorcraft - A Survey," Journal of Aircraft, Vol. 36, No. 1, jan-feb 1999, pp. 176-189.

${ }^{4}$ Ganguli, R., "Survey of Recent Developments in Rotorcraft Design Optimization," Journal of Aircraft, Vol. 41, No. 3, May-June 2004, pp. 493-510.

${ }^{5}$ Friedmann, P. and Millott, T., "Vibration Reduction in Rotorcraft Using Active Control: A Comparison of Various Approaches," Journal of Guidance, Control, and Dynamics, Vol. 18, No. 4, July-August 1995, pp. 664-673.

${ }^{6}$ Patt, D., Liu, L., and Friedmann, P. P., "Rotorcraft Vibration Reduction and Noise Prediction Using a Unified Aeroelastic Response Simulation," Journal of the American Helicopter Society, Vol. 50, No. 1, January 2005.

${ }^{7}$ Schmit, L. and Miura, H., Concepts for Efficient Structural Synthesis, NASA CR-2552, 1976.

${ }^{8}$ Yuan, K. A. and Friedmann, P., Aeroelasticity and Structural Optimization of Composite Helicopter Rotor Blades with Swept Tips, NASA CR 4665, May 1995.

${ }^{9}$ Wujek, B. and Renaud, J., "Improved Trust Region Model Management for Approximate Optimization," Advances in Design Automation, Atlanta, Georgia, September 13-16 1998, Paper No. DETC98/DAC-5616.

${ }^{10}$ Alexandrov, N., Dennis Jr., J., Lewis, R., and Torczon, V., "A Trust Region Framework for Managing the use of Approximate Models in Optimization," Structural Optimization, Vol. 15, No. 1, 1998, pp. 16-23.

${ }^{11}$ Ganguli, R., "Optimal Design of a Low Vibration Helicopter Rotor Using Response Surface Approximation," Journal of Sound and Vibration, Vol. 258, No. 2, 2002, pp. 327-344.

${ }^{12}$ Jones, D., Schonlau, M., and Welch, W., "Efficient Global Optimization of Expensive Black-Box Functions," Journal of Global Optimization, Vol. 13, 1998, pp. 455-492.

${ }^{13}$ Sóbester, A., Leary, S., and Keane, A., "A Parallel Updating Scheme for Approximating and Optimizing High Fidelity Computer Simulations," Structural and Multidisciplinary Optimization, Vol. 27, 2004, pp. 371-383.

${ }^{14}$ Booker, A., Dennis Jr., J., Frank, P., Serafini, D., Torczon, V., and Trosset, M., "A Rigorous Framework for Optimization of Expensive Functions by Surrogates," Structural Optimization, Vol. 17, 1999, pp. 1-13.

${ }^{15}$ Shultz, L., Panda, B., Tarzanin, F., Derham, R., Oh, B., and Dadone, L., "Interdisciplinary Analysis for Advanced Rotors - Approach, Capabilities, and Status," American Helicopter Society Aeromechanics Specialists Conference, 1994, PS 4-1-4-15.

${ }^{16}$ Millott, T. A. and Friedmann, P. P., Vibration Reduction in Helicopter Rotors Using an Actively Controlled Partial Span Trailing Edge Flap Located on the Blade, NASA CR 4611, June 1994.

${ }^{17}$ Yuan, K. A. and Friedmann, P. P., Aeroelastic and Structural Optimization of Composite Helicopter Rotor Blades with Swept Tips, NASA CR 4665, June 1995.

${ }^{18}$ Myrtle, T. F. and Friedmann, P. P., "Application of a New Compressible Time Domain Aerodynamic Model to Vibration Reduction in Helicopters Using an Actively Controlled Flap," Journal of the American Helicopter Society, Vol. 46, No. 1, Jan. 2001, pp. 32-43.

${ }^{19}$ de Terlizzi, M. and Friedmann, P. P., "Active Control of BVI Induced Vibrations Using a Refined Aerodynamic Model and Experimental Correlation," Proceedings of the 55th Annual Forum of the American Helicopter Society, Montreal, Canada, May 1999, pp. 599-615.

${ }^{20}$ Depailler, G. and Friedmann, P. P., "Reductions of Vibrations Due to Dynamic Stall in Helicopters Using an Actively Controlled Flap," Proceedings of the 43rd AIAA/ASME/ASCE/AHS/ACS Structures, Structural Dynamics and Materials Conference, Denver, CO, April 2002, AIAA Paper No. 2002-1431.

${ }^{21}$ Liu, L., Friedmann, P. P., and Patt, D., "Simultaneous Vibration and Noise Reduction in Rotorcraft - Practical Implementation Issues," Proceedings of the 46th AIAA/ASME/ASCE/AHS/ACS Structures, Structural Dynamics and Materials Conference, Austin, TX, April 2005.

${ }^{22}$ Myrtle, T. F., Development of an Improved Aeroelastic Model for the Investigation of Vibration Reduction in Helicopter Rotors using Trailing Edge Flaps, Ph.D. thesis, University of California, Los Angeles, 1998.

${ }^{23}$ Patt, D., Liu, L., and Friedmann, P. P., "Rotorcraft Vibration Reduction and Noise Prediction Using a Unified Aeroelastic Response Simulation," Journal of the American Helicopter Society, Jan. 2005.

${ }^{24}$ Johnson, W., CAMRAD/JA - A Comprehensive Analytical Model of Rotorcraft Aerodynamics and Dynamics, Vol I. Theory Manual, Johnson Aeronautics, Palo Alto, CA, 1988.

${ }^{25}$ Johnson, W., CAMRAD/JA - A Comprehensive Analytical Model of Rotorcraft Aerodynamics and Dynamics, Vol II. Users' Manual, Johnson Aeronautics, Palo Alto, CA, 1988.

${ }^{26}$ de Terlizzi, M. and Friedmann, P. P., "Aeroelastic Response of Swept Tip Rotors Including the Effects of BVI," Proceedings of the 55th Annual Forum of the American Helicopter Society, Washington D.C., May 1998, pp. 644-663.

${ }^{27}$ Celi, R. and Friedmann, P., "Structural Optimization with Aeroelastic Constraints of Rotor Blades with Straight and Swept Tips," AIAA Journal, Vol. 28, No. 5, 1990, pp. 928-936.

${ }^{28}$ Sacks, J., Welch, W., Mitchell, T., and Wynn, H., "Design and Analysis of Computer Experiments," Statistical Science, Vol. 4, No. 4, 1989, pp. 409-435.

${ }^{29}$ Simpson, T., Booker, A., Ghosh, D., Giunta, A., Koch, P., and Yang, R., "Approximation Methods in Multidisciplinary Analysis and Optimization: A Panel Discussion," 9th AIAA/ISSMO Symposium on Multidisciplinary Analysis 83 Optimization: Approximation Methods Panel, Atlanta, Georgia, September 2-4 2002, pp. 1-16.

${ }^{30}$ McKay, M., Beckman, R., and Conover, W., "A Comparison of Three Methods for Selecting Values of Input Variables in the Analysis of Output from a Computer Code," Technometrics, Vol. 21, No. 2, May 1979, pp. 239-245. 
${ }^{31}$ Queipo, N., Haftka, R., Shy, W., Goel, T., Vaidyanathan, R., and Tucker, P., "Surrogate-Based Analysis and Optimization," Progress in Aerospace Sciences, Vol. 41, 2005, pp. 1-28.

${ }^{32}$ Jin, R., Chen, W., and Sudjianto, A., "An Efficient Algorithm for Constructing Optimal Design of Computer Experiments," ASME Design AUtomation Conference, Chicago, IL, September 2-6 2003, DETC-DAC48760.

${ }^{33}$ Jin, R., Chen, W., and Simpson, T., "Comparative Studies of Metamodeling Techniques Under Multiple Modeling Criteria," Structural and Multidisciplinary Optimization, Vol. 23, 2001, pp. 1-13.

${ }^{34}$ Sasena, M., Flexibility and Efficiency Enhancements for Constrained Global Optimization with Kriging Approximations, Ph.D. thesis, University of Michigan, 2002.

${ }^{35}$ Simpson, T., Peplinski, D., Koch, P., and Allen, J., "Metamodels for Computer-based Engineering Design: Survey and recommendations," Engineering with Computers, Vol. 17, 2001, pp. 129-150.

${ }^{36}$ Schonlau, M., Computer Experiments and Global Optimization, Ph.D. thesis, University of Waterloo, 1997.

${ }^{37}$ Jones, D., "The DIRECT global optimization algorithm," Encyclopedia of Optimization, Vol. 1, 2001, pp. 431-440.

${ }^{38}$ Liu, L., Patt, D., and Friedmann, P. P., "Simultaneous Vibration and Noise Reduction in Rotorcraft Using Aeroelastic Simulation," Proceedings of the 60th American Helicopter Society Annual Forum, Baltimore, MD, June 2004.

${ }^{39} \mathrm{Lim}, \mathrm{J}$. and Chopra, I., "Aeroelastic Optimization of a Helicopter Rotor Using an Efficient Sensitivity Analysis," Journal of Aircraft, Vol. 28, No. 1, January 1991, pp. 29-37.

${ }^{40}$ Friedmann, P. and Shanthakumaran, P., "Optimum Design of Rotor Blades for Vibration Reduction in Forward Flight," Journal of the American Helicopter Society, Vol. 29, No. 4, 1984, pp. 70-80.

${ }^{41}$ Lim, J. and Chopra, I., "Aeroelastic Optimization of a Helicopter Rotor," Journal of the American Helicopter Society, Vol. 34, No. 1, 1989, pp. 55-62.

${ }^{42}$ Koch, P., Evans, J., and Powell, D., "Interdigitation for Effective Design Space Exploration using iSIGHT," Structural and Multidisciplinary Optimization, Vol. 23, No. 2, 2002, pp. 111-126.

${ }^{43}$ Liu, L., BVI Induced Vibration and Noise Alleviation by Active and Passive Approaches, Ph.D. thesis, University of Michigan, 2005. 